\title{
Experimental setup for camera-based measurements of electrically and optically stimulated luminescence of silicon solar cells and wafers
}

David Hinken, Carsten Schinke, Sandra Herlufsen, Arne Schmidt, Karsten Bothe, and Rolf Brendel

Citation: Review of Scientific Instruments 82, 033706 (2011);

View online: https://doi.org/10.1063/1.3541766

View Table of Contents: http://aip.scitation.org/toc/rsi/82/3

Published by the American Institute of Physics

\section{Articles you may be interested in}

Photoluminescence imaging of silicon wafers

Applied Physics Letters 89, 044107 (2006); 10.1063/1.2234747

Photographic surveying of minority carrier diffusion length in polycrystalline silicon solar cells by electroluminescence

Applied Physics Letters 86, 262108 (2005); 10.1063/1.1978979

Diffusion lengths of silicon solar cells from luminescence images

Journal of Applied Physics 101, 123110 (2007); 10.1063/1.2749201

Series resistance imaging of solar cells by voltage dependent electroluminescence

Applied Physics Letters 91, 182104 (2007); 10.1063/1.2804562

Bulk minority carrier lifetimes and doping of silicon bricks from photoluminescence intensity ratios Journal of Applied Physics 109, 083111 (2011); 10.1063/1.3575171

Advanced luminescence based effective series resistance imaging of silicon solar cells Applied Physics Letters 93, 202102 (2008); 10.1063/1.2982588

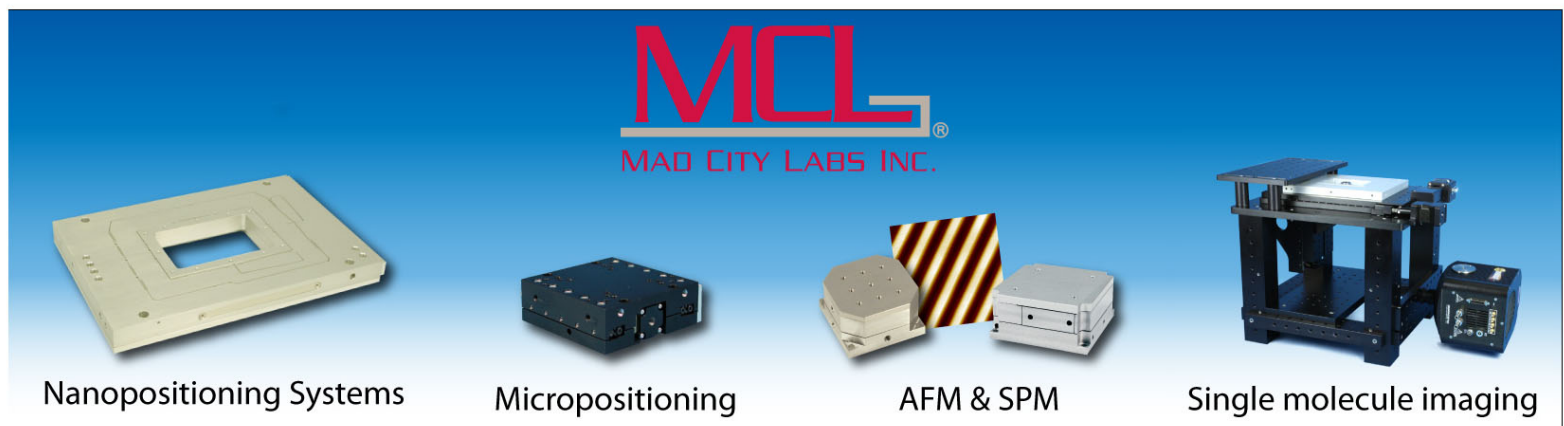




\title{
Experimental setup for camera-based measurements of electrically and optically stimulated luminescence of silicon solar cells and wafers
}

\author{
David Hinken, ${ }^{\text {a) }}$ Carsten Schinke, Sandra Herlufsen, Arne Schmidt, Karsten Bothe, and \\ Rolf Brendel \\ Institute for Solar Energy Research Hamelin (ISFH), Am Ohrberg 1, 31860 Emmerthal, Germany
}

(Received 7 November 2010; accepted 22 December 2010; published online 3 March 2011)

\begin{abstract}
We report in detail on the luminescence imaging setup developed within the last years in our laboratory. In this setup, the luminescence emission of silicon solar cells or silicon wafers is analyzed quantitatively. Charge carriers are excited electrically (electroluminescence) using a power supply for carrier injection or optically (photoluminescence) using a laser as illumination source. The luminescence emission arising from the radiative recombination of the stimulated charge carriers is measured spatially resolved using a camera. We give details of the various components including cameras, optical filters for electro- and photo-luminescence, the semiconductor laser and the four-quadrant power supply. We compare a silicon charged-coupled device (CCD) camera with a back-illuminated silicon CCD camera comprising an electron multiplier gain and a complementary metal oxide semiconductor indium gallium arsenide camera. For the detection of the luminescence emission of silicon we analyze the dominant noise sources along with the signal-to-noise ratio of all three cameras at different operation conditions. (C) 2011 American Institute of Physics. [doi:10.1063/1.3541766]
\end{abstract}

\section{INTRODUCTION}

Luminescence imaging was first reported in 1963 by Uchida $^{1}$ who used an infrared-sensitive image converter tube to show spatially resolved the radiative recombination from forward biased silicon p-n junctions. An improved experimental setup was used by Penner ${ }^{2}$ in 1988 connecting a computer-controlled video camera tube to the infrared image converter. His camera-based approach allowed for a quick and automatic inspection of large area semiconductor devices such as silicon solar cells. Only two years later, in 1990, Livescu et $a l^{3}$ reported on a real-time photoluminescence (PL) imaging system exemplarily used for the analysis of GaAs/AlGaAs p-i-n quantum well modulators and InP substrates. In this setup, a video camera tube is used for visible light and a lead sulfide camera for infrared light.

For the analysis of large area crystalline silicon solar cells electroluminescence imaging was brought to the attention of the photovoltaic community by Fuyuki et al. ${ }^{4}$ in 2005. Fuyuki et al. demonstrated that the electroluminescence emission of silicon solar cells is directly detectable with commercially available silicon charge-coupled device (CCD) cameras without the need of infrared image converters. Using optical instead of electrical excitation Trupke et al. ${ }^{5}$ utilized photoluminescence imaging as a versatile tool for solar cells and wafer characterization under realistic operating conditions comprising simultaneous optical and electrical excitation.

Besides the extensive qualitative information included in luminescence images, numerous evaluation procedures for a quantitative analysis ${ }^{6}$ were introduced. For silicon solar cells techniques for the local determination of voltages, ${ }^{7,8}$ series resistances, ${ }^{9-13}$ diffusion lengths, ${ }^{14-17}$ saturation current densities, ${ }^{13,18,19}$ and shunt resistances ${ }^{20,21}$ are to be

a)Electronic mail: hinken@isfh.de. mentioned. All of these techniques take advantage of measuring the solar cell at different working points (voltage and/or illumination), by measuring only spectral fractions of the luminescence using optical filters or by analyzing the lateral distributions.

Regarding silicon wafers, images of the effective carrier lifetime were obtained by calibration with other lifetime measurement techniques such as infrared lifetime mapping/carrier density imaging, ${ }^{22,23}$ quasi-steadystate photoluminescence, ${ }^{24,25}$ and by photoconductance measurements. ${ }^{26-29}$ Without any external calibration the effective lifetime was determined with the knowledge of all relevant parameters of the used setup ${ }^{17}$ and from the time dependent luminescence emission for a modulated optical excitation. ${ }^{30,31}$

In this work, we present the luminescence imaging setup developed within the last years in our laboratory. We not only report on the relevant equipment but also analyze the components of the setup in detail. For a quantitative analysis of the luminescence emission the camera is of specific importance. For this reason, we compare a silicon CCD camera with a back-illuminated silicon CCD camera comprising an electron multiplier (EM) gain and a complementary metal oxide semiconductor (CMOS) indium gallium arsenide (In$\mathrm{GaAs}$ ) camera. The dominant noise sources along with the signal-to-noise (SNR) ratio are analyzed for all three cameras. Moreover, for the detection of the luminescence emission of silicon, the signal-to-noise ratio of these cameras is calculated and experimentally confirmed with respect to the total measurement time and the total luminescence photon flux.

\section{EXPERIMENTAL SETUP}

The experimental setup shown in Fig. 1 comprises a measurement chuck, a solar cell contacting unit, a bipolar power 


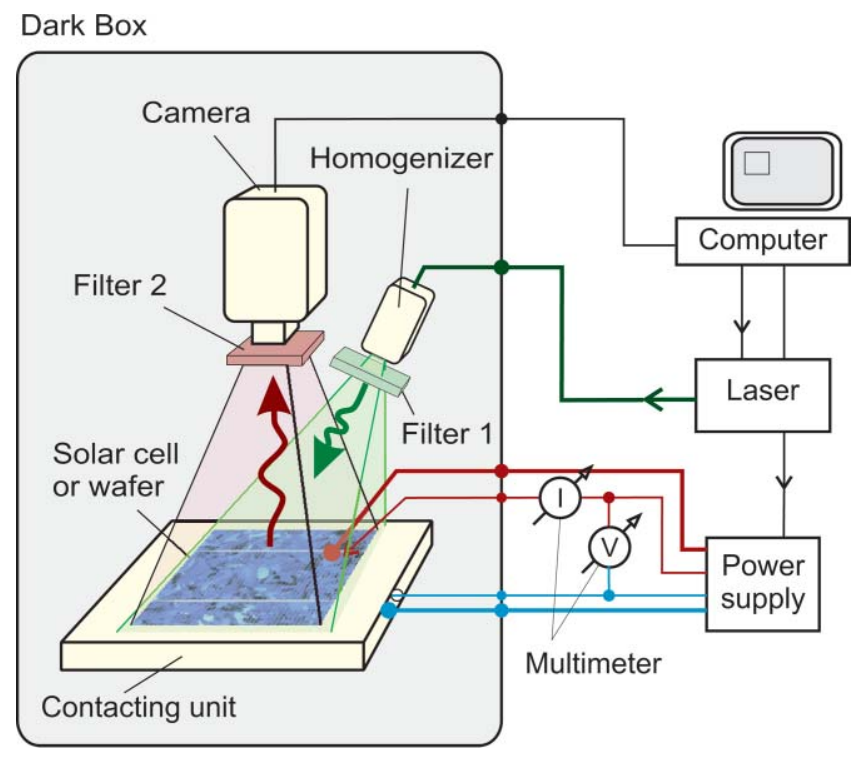

FIG. 1. (Color online) Setup for spatially resolved luminescence measurements.

supply, a semiconductor laser, a homogenization optics, a camera, various filters, two multimeters, a vacuum pump, a water thermostat, a linear unit, and a computer.

To avoid the detection of ambient light, the camera, the measurement chuck, the solar cell contacting unit and the homogenization optics are mounted in a dark box $(1290 \mathrm{~cm}$ $\times 700 \mathrm{~cm} \times 690 \mathrm{~cm})$. All metallic parts of this box are blackened to minimize interference due to multiple reflected light within the box. The side-walls of the box consists of black polyvinyl chloride plastic (SIMONA Simocel-Color black $6 \mathrm{~mm}$ ). Measurements show that the hemispherical reflectance of this material is smaller than $6 \%$ in the wavelength range of 400-1200 $\mathrm{nm}$. Any objects inside the box have to be carefully examined to not disturb the measured luminescence emission of the solar cell or the wafer. We measure (optically excited by the semiconductor laser) weak parasitic luminescence emission from sheets of paper, strong emission from pertinax that is used for the isolation of the sense and the current wires and strong emission from cables and connectors (particularly if coated with a red color).

The silicon sample under test, a solar cell or a wafer, is mounted on a vacuum chuck (custom-made product, ISFH) for samples of up to $20 \times 20 \mathrm{~cm}^{2}$. The chuck is temperaturecontrolled by a closed-loop water thermostat (Haake, DC30$\mathrm{K} 15$ ), thus offering a temperature range from approximately 5 to $85^{\circ} \mathrm{C}$. A small PT1000 temperature sensor is mounted within the chuck's surface. The temperature sensor is pressed to the rear of the sample by a small spring and has no direct contact with the measurement chuck. Thus, the measured temperature corresponds directly to the temperature of the sample. For silicon wafers, the pattern of the measurement chuck may appear in the captured luminescence image due to a laterally inhomogeneous surface reflectance. In this case, we place the wafer on a thin, black, plastic pad and accept the disadvantage of reduced temperature stabilization.

For solar cells, a four-quadrant voltage and current power supply (Kepco, BOP36-28MG) controls the applied voltage.
Depending on the operating conditions electrical current is extracted from or fed into the solar cell. To eliminate errors due to lead resistances we use a four-point contacting scheme with remote sensing. The busbars of the solar cell under test are contacted by up to three bars containing spring loaded needles. The brass chuck itself is used as the rear contact. One needle in each bar and one in the chuck are isolated from the others to sense the voltage (sense contacts).

To determine the applied voltage $V_{\text {appl }}$ at the solar cell we use a multimeter (Keithley, Model 2000) connected to the sense wires. Regarding current measurements, one of the current carrying wires contains a small four-wire resistance $R_{\mathrm{m}}=100 \mathrm{~m} \Omega$ in series. The voltage drop $\Delta V$ at $R_{\mathrm{m}}$ is measured with a second multimeter (Keithley, Model 2000). The current fed into or extracted from the solar cell follows directly from Ohm's law.

For the monochromatic illumination we use a gallium arsenide (GaAs) diode laser (Jenoptik unique-mode, JUM30k/400/20) which exhibits a central wavelength (CWL) of $809 \mathrm{~nm}$. For silicon, this CWL corresponds to an absorption length of $\sim 13 \mu \mathrm{m}$. The maximum continuous wave output power of the laser is $30 \mathrm{~W}$. A beam homogenizer (custommade product, Bayerisches Laserzentrum $\mathrm{GmbH}$ ) based on microlenses shapes the fiber output beam to a square flattop. For the optimum working distance of $75 \mathrm{~cm}$ an area of $16 \times 16 \mathrm{~cm}^{2}$ is illuminated at an angle of $15^{\circ}$ with an homogeneity of $\pm 10 \%$. Considering the losses of the fiber and the optics $(\sim 40 \%)$, we reach an equivalent photogeneration rate of up to two-thirds sun.

The camera detecting the luminescence emission is mounted directly above the contacting unit. We have two silicon (Si) charge-coupled device cameras (PCO Sensicam QE and Hamamatsu C9100-13) and one InGaAs complementary metal-oxide semiconductor camera (Xenics Cheetah) at our disposal. All relevant parameters of these cameras are listed in Table I. The different electron fluxes and noise sources were determined in our laboratory. The measured signal electron flux corresponds to an electroluminescence spectrum emitted from a solar cell operating at an applied voltage of $521 \mathrm{mV}$. To be able to compare the signal electron flux for different cameras equal measurement conditions (same optics, same position of the solar cell's image within the sensor, same luminescence intensity) were maintained. For the Sensicam QE camera a $2 \times 2$ binning was used to account for the larger image sensor.

The main difference between $\mathrm{Si}$ and $\mathrm{InGaAs}$ cameras is their sensitivity to different wavelength ranges as shown in Fig. 2. InGaAs absorbs photons ranging from 900 to $1700 \mathrm{~nm}$ and thus covers the whole range of the luminescence emission from silicon. In contrast, $\mathrm{Si}$ is better suited for the visible range and its quantum efficiency reduces significantly for wavelengths larger than $1000 \mathrm{~nm}$. Note that we focus in this paper on the detection of band-to-band luminescence emission only. Additional light emission (see, for example, Ref. 32 and papers cited in there) may be found in the sub-band-gap range between 1400 and $1700 \mathrm{~nm}$ for solar cells and wafers and in the visible range for reverse-biased solar cells. Figure 2 demonstrates that the sub-band-gap light detection requires an InGaAs detector and the visible light emission a Si detector. 
TABLE I. Camera parameters.

\begin{tabular}{|c|c|c|c|}
\hline & Sensicam QE (Si) & C9100-13 (Si) & Cheetah (InGaAs) \\
\hline Manufacturer & PCO & Hamamatsu & Xenics \\
\hline Operation mode 1 & Lowlight, high gain & Normal-CCD (nrm) & Low gain (LG) \\
\hline Operation mode 2 & $\ldots$ & EM-Gain 1200 (EM) & High gain $(\mathrm{HG})$ \\
\hline Sensor & $\begin{array}{l}\text { Sony ICX285-AL Interline } \\
\text { CCD }\end{array}$ & $\begin{array}{l}\text { E2V CCD97 Back-thinned } \\
\text { frame transfer with on-chip } \\
\text { amplification (EM-Gain) }\end{array}$ & XFPA-1.7-640-TE1-V4 Xenics-built \\
\hline Sensor size $\left(\mathrm{mm}^{2}\right)$ & $6.6 \times 8.8(2 / 3$ in $)$ & $8.2 \times 8.2$ & $12.8 \times 10.2$ \\
\hline Pitch size $(\mathrm{mm})$ & 6.45 & 16 & 20 \\
\hline No. of pixels & $1376 \times 1040$ & $512 \times 512$ & $640 \times 512$ \\
\hline Sensor cooling $\left({ }^{\circ} \mathrm{C}\right)$ & -12 & -65 & 4 \\
\hline Full well capacity $N_{\mathrm{FW}}\left(e^{-}\right)$ & 18000 & 370000 & 1250000 \\
\hline $\mathrm{A} / \mathrm{D}$ conversion (bit) & 12 & 16 & 14 \\
\hline Conversion factor $\left(e^{-} / D U\right)$ & 2 & $1.4(\mathrm{nrm})$ and $5.8(\mathrm{EM})$ & $76.29(\mathrm{LG})$ and $5.34(\mathrm{HG})$ \\
\hline Dark electron flux $\Phi_{\text {drk }}\left(e^{-} / s / p x\right)$ & 0.64 & 0.01 & $280000(\mathrm{LG})$ and $220000(\mathrm{HG})$ \\
\hline Signal electron flux $\Phi_{s}\left(e^{-} / s / p x\right)$ & $0.388 \times 10^{-3}$ & $1.833 \times 10^{-3}$ & $0.833(\mathrm{LG})$ and $0.666(\mathrm{HG})$ \\
\hline Readout noise $\sigma_{\mathrm{ro}}\left(e^{-} / p x\right)$ & 4.8 & $16.8(\mathrm{nrm})$ and $215(\mathrm{EM})$ & $122(\mathrm{LG})$ and $104(\mathrm{HG})$ \\
\hline Temperature noise $\sigma_{\mathrm{tmp}}\left(e^{-} / p x\right)$ & 0 & 0 & $620(\mathrm{LG})$ and $62(\mathrm{HG})$ \\
\hline
\end{tabular}

All three cameras feature a C-mount (corresponding to a flange focal distance of $b=17.526 \mathrm{~mm}$ ) as the lens mount. We use the same lens [Pentax, C2514-M (KP)] for all three cameras. The aperture of this lens is 1.4 and the focal length $\mathrm{f}=25 \mathrm{~mm}$. To cover the whole area of the sensor with the image of the solar cell the distance $g$ between the solar cell and the lens is adjustable. Therefore, the camera is mounted on a linear unit which changes $g$ between 0 and $80 \mathrm{~cm}$. The minimum object distance of this lens is $25 \mathrm{~cm}$, but using close-up lens rings smaller distances are achievable.

Optical filters are necessary for quantitative measurements of the luminescence emission. For EL measurements we use a longpass filter (Semrock-Razoredge 830) to avoid the detection of stray light. Due to the dark box this longpass filter is not really required but is useful when focusing the camera with the box open. The filter choice is much more important for PL measurements of silicon wafers or solar cells since reflected excitation light has to be suppressed. Figure 3 shows the spectra of the laser and the transmittances of the filters that we use in our PL setup.

Filter 1 in Fig. 3 is a bandpass (custom product, bkInterferenzoptik) with a transmittance higher than $70 \%$ in the wavelength range from 805 to $815 \mathrm{~nm}$ and an optical density higher than eight outside this range. The bandpass filter is positioned at the exit of the homogenizer (see Fig. 1) and blocks the emission of the laser at long wavelengths. This is necessary since the sharp laserline is weakly superimposed by a broad spectrum due to spontaneous emissions from the GaAs laser diode (the amplified spontaneous emission spectrum). In order not to damage the filter, it is important to mount the filter at the exit of the homogenization optics where the laser

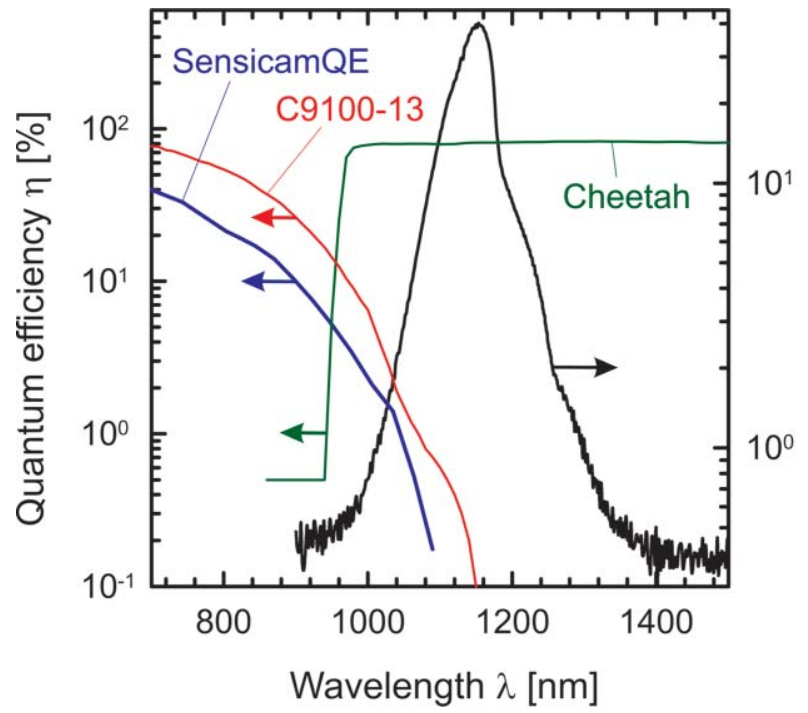

FIG. 2. (Color online) Quantum efficiencies (manufacturer information) of the used cameras (Sensicam QE, Hamamatsu C9100-13 and Xenics Cheetah) and a luminescence spectrum of a monocrystalline silicon solar cell.

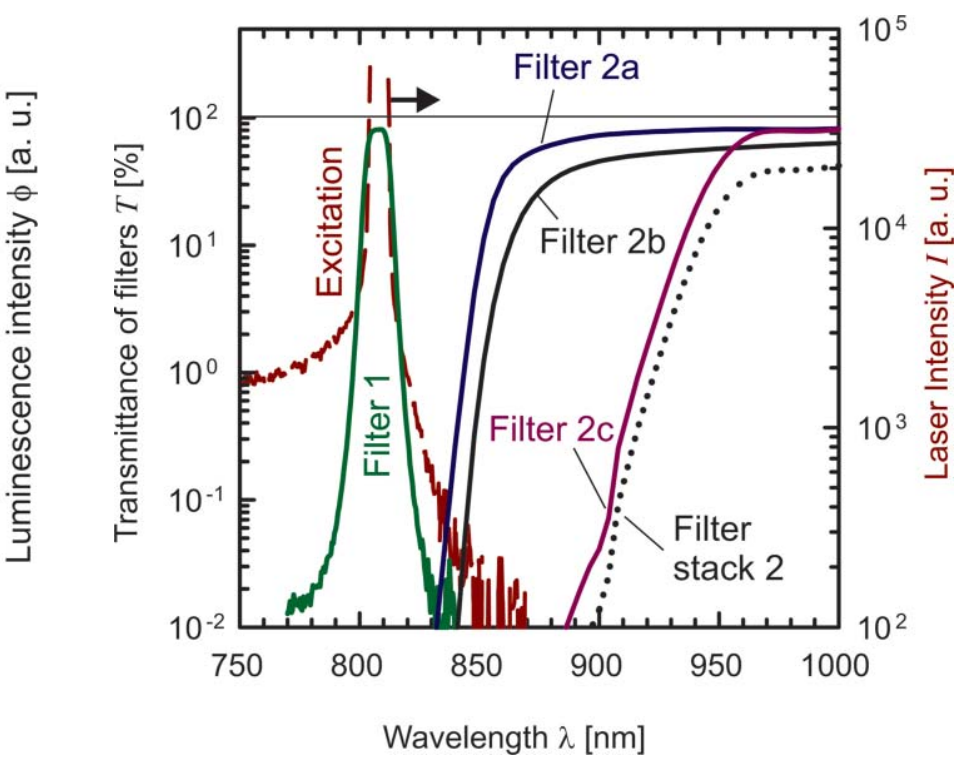

FIG. 3. (Color online) Laser spectrum and transmittances of the filters used for photoluminescence imaging. 
output beam is already widened and the intensity is thus reduced.

The filter stack 2 in Fig. 3 consists of several longpass filters positioned in front of the camera (see Fig. 1). It blocks the reflected laser light while exhibiting a high transmission for the luminescence photons. For PL measurements with the InGaAs-camera the requirements for this stack are low since the InGaAs-camera has a small quantum efficiency at the central wavelength of the laser (see Fig. 2). Hence, a standard $1000 \mathrm{~nm}$ longpass filter (Thorlabs FEL1000) is sufficient in most cases. For PL measurements with Si-cameras, which feature a high quantum efficiency at the laser's central wavelength, a filter stack with an extremely high optical density to block the reflected laser light is crucial. A $20 \mathrm{~mm}$ thick absorbing glass filter (RG850, Schott-Glass) provides this optical density at $808 \mathrm{~nm}$ (filter $2 \mathrm{~b}$ in Fig. 3). However, due to the strong absorption of photons within the RG850 filter the filter itself emits light ${ }^{33}$ because it contains nanocrystallites of II-IV semiconducting compounds. ${ }^{34}$ Even though in most cases this parasitic light emission is negligibly small it may disturb the measurement significantly for PL measurements at solar cells or wafers with very low luminescence emission.

We therefore place two additional interference filters (filters $2 \mathrm{a}$ and $2 \mathrm{c}$ ) on both sides of the RG850 (filter $2 \mathrm{~b}$ ). Filter $2 \mathrm{a}$, positioned underneath the RG850, reflects the vast majority of the laser light back into the setup and thus decreases the light intensity entering into the RG850. Filter 2c, positioned between the RG850 and the camera, blocks the parasitic light generated within the RG850. Filters 2a and $2 \mathrm{c}$ are custom-made longpass filters (bk-Interferenzoptik, 2a: $856 \mathrm{~nm}, 2 \mathrm{c}: 930 \mathrm{~nm}$ ) which exhibit a high blocking (2a: OD9, 2c: OD5) for short wavelengths and a high transmission (90\%) for longer wavelengths. The transmittances of these filters are shown in Fig. 3 together with the resulting transmittance of the filter stack 2 (comprising filter $2 \mathrm{a}, 2 \mathrm{~b}$, and $2 \mathrm{c}$ ).

\section{DATA ACQUISITION}

The whole data acquisition process is controlled by a computer software. This software was developed at the ISFH using the programming language LABVIEW (National Instruments). The power supply and the multimeters are controlled via a general purpose interface bus (GPIB) and the laser, the linear unit and the water thermostat via an RS232 interface. The two Si-cameras are connected to the computer using proper peripheral component interconnect (PCI) cards from the manufacturer and the Cheetah camera is connected via an Ethernet interface. To prepare the setup for the capturing of luminescence images, we firstly mount the sample under test on the measurement chuck. Then, we adjust the camera's height to use the full sensor size for the image of the solar cell and focus the camera to the luminescence emission. Finally, the parameters characterizing the measurement conditions are set in the data acquisition program. A batch mode may be used to automatically measure luminescence images at a sequence of measurement conditions.

After this preparations the data acquisition is fully automatically. The data acquisition program first initializes all devices and, in case of a solar cell, performs a contact check.
Then, the power supply and the laser are switched from standby to operating mode to stimulate the luminescence emission. While the camera is capturing the luminescence image the temperature of the sample is measured and in case of a solar cell additionally the applied voltage and current. After exposure, the resulting luminescence image is read out from the camera and the power supply and or the laser are put back into standby mode. This procedure is repeated with the laser and the power supply in standby mode (no luminescence stimulation) to measure the so-called dark image. The dark image is automatically subtracted from the luminescence image to account for remaining stray light and any camera offsets.

To improve the signal-to-noise ratio the data acquisition process is repeated as often as required. After averaging $M$ times, the signal for each pixel

$$
N_{\mathrm{s}}=\frac{1}{M} \sum_{i}^{M} N_{\mathrm{s}, i}
$$

and the standard deviation

$$
\sigma_{\mathrm{s}}=\sqrt{\frac{1}{M} \sum_{i}^{M}\left(N_{\mathrm{s}}-N_{\mathrm{s}, i}\right)^{2}}
$$

is calculated. The signal-to-noise ratio becomes

$$
\mathrm{SNR}=\frac{\mathrm{N}_{\mathrm{s}}}{\mathrm{u}}=\frac{\mathrm{N}_{\mathrm{s}}}{\sigma_{\mathrm{s}}} \sqrt{\mathrm{M}},
$$

where $u=\sigma_{\mathrm{s}} / \sqrt{M}$ is the standard deviation of the mean.

A nonuniformity correction (NUC) of the acquired images is necessary to correct for inhomogeneities of the lateral quantum efficiency of the camera sensor and inhomogeneous transmission of the lenses and filters. Therefore, the luminescence image is divided by the NUC image which is captured using a homogeneous luminescence light source. Our approach is to use the same focus settings as for the real measurement but with the camera as close as possible (typically $10 \mathrm{~cm}$ ) above a monocrystalline silicon solar cell. In this position the solar cell is completely out of focus for the camera and illuminates the sensor homogeneously. We capture the NUC image with an exposure time corresponding to half full well capacity and repeat the measurement at least 100 times to lower the dark and photon noise.

Further correction of the images is required if defect pixels exist. Defect pixels on the sensor have no signal at all (dead pixel) or too much signal (hot pixel) due to a high dark current. These defect pixels have to be neglected in any quantitative analysis. Most of the defect pixels are at a fixed position and can thus be identified easily and corrected for by assigning the average value of the neighboring pixels.

\section{EXEMPLARY LUMINESCENCE IMAGES}

The presented experimental setup allows to measure the luminescence emission of solar cells and wafers at different operation conditions. 


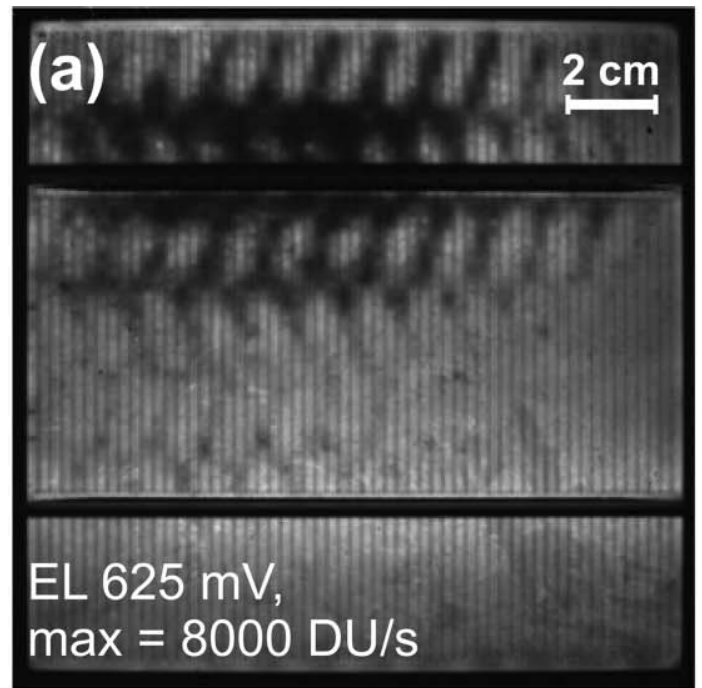

\section{$\max$}
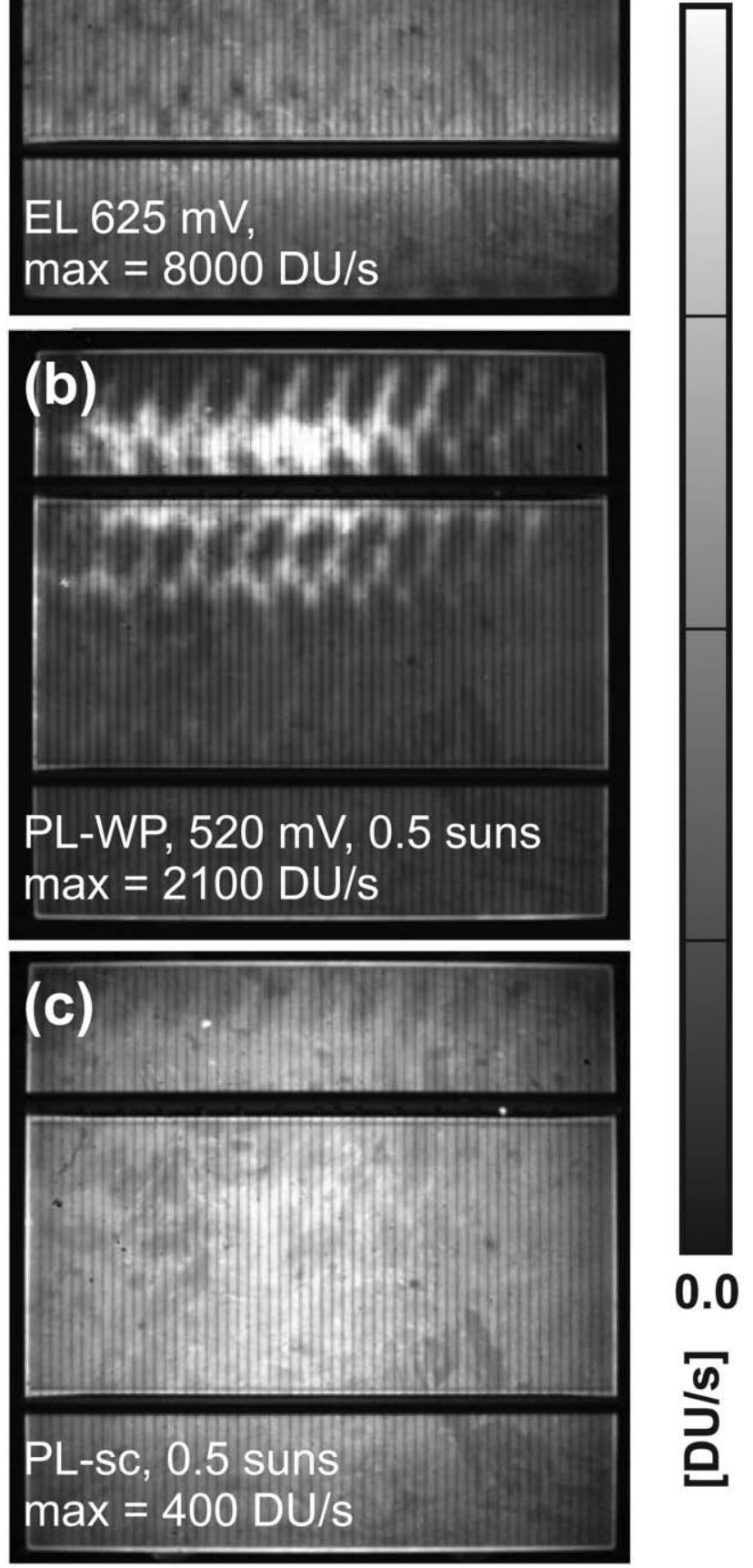

FIG. 4. Luminescence emission of a multicrystalline solar cell at different working points, captured with the Cheetah camera.

Figure 4 shows three images of the luminescence emission of a poorly processed multicrystalline silicon solar cell. The total measurement time for all images is $20 \mathrm{~s}$. The solar cell in Fig. 4(a) is in complete darkness and held at an applied voltage of $625 \mathrm{mV}$ (electroluminescence). In Figs. 4(b) and 4(c) the solar cell is illuminated by the laser at 0.5 suns. While all current is extracted from the solar cell in Fig. 4(c), which means short-circuit conditions $\left(V_{\text {appl }}=0\right)$, the solar cell in Fig. 4(b) is held at a specific working point (PL-WP).

Figure 5 shows the effective lifetime image of a multicrystalline silicon wafer. The lifetime values were obtained by a PL measurement calibrated with steadystate photoconductance. ${ }^{29}$ The PL image is captured with the Hamamatsu camera at an illumination intensity of 1.5 suns and a total measurement time of $3.5 \mathrm{~s}$. The high illumination intensity could be achieved because only a region of $8 \times 8 \mathrm{~cm}^{2}$ was measured.

The images in Figs. 4 and 5 demonstrate high-quality images that are full of information on the strengths and weaknesses of the investigated samples. In this paper, we will only give a short interpretation of these two images.

Clearly visible in Fig. 4 is a locally increased series resistance probably induced by the transport band in the solar cell's firing process. While this series resistance appears as a decreased luminescence signal in the EL image [see Fig. 4(a)], it leads to an increased signal in the PLWP image [see Fig. 4(b)]. ${ }^{5}$ In contrast, the PL-sc image does hardly show any pattern of the local series resistance. This becomes understandable if we consider the shape of the illuminated current-voltage characteristics where a small or moderate series resistance does not have any impact on the short circuit current. The PL-sc image shows the diffusion limited carriers, ${ }^{9,35}$ which cannot be extracted from the solar cell's base even at short-circuit conditions. The recombination properties of the multicrystalline material are visible in all three images shown in Fig. 4. Shunts would appear as a decreased luminescence signal in the EL and in the PL-wp image. We do not find obvious shunts. Unless the shunt resistance is very small it will not appear in the PL-sc image.

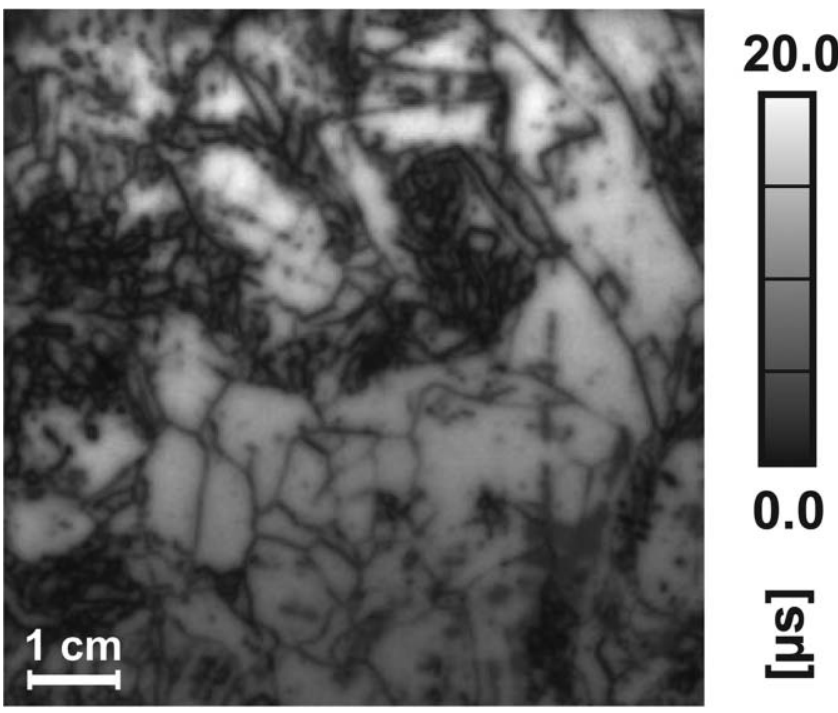

FIG. 5. Lifetime image of a multicrystalline wafer captured with the Hamamatsu camera. The effective lifetime was calibrated with steady-state photoconductance (see Ref. 29). The total measurement time for the PL image is $3.5 \mathrm{~s}$ and the spatial resolution is $155 \mu \mathrm{m}$. 
The image shown in Fig. 5 of a silicon wafer shows the local effective lifetime. Clearly visible are the grains and the grain boundaries of the multicrystalline material. The grains appear in Fig. 5 as quite homogeneous regions with lifetime values around $15 \mathrm{~ms}$. These regions are separated by grain boundaries which correspond to dark lines with a high recombination activity. In addition, areas with a high density of dendritic lines are found which originate from recombination active intragrain defects such as stacking faults and dislocations. These crystal irregularities are recombination active due to dangling bonds or decorations with recombination active impurities. ${ }^{36}$

\section{CAMERA NOISE ANALYSIS}

We carry out a detailed noise analysis for the different cameras used in our system. This noise analysis focuses on the analytical determination of the signal-to-noise ratio

$$
\mathrm{SNR}=\frac{N_{\mathrm{s}}}{u},
$$

which depends on the number of generated signal electrons $N_{\mathrm{s}}$ and its standard deviation of the mean $u$. Based on this noise analysis we deduce sets of camera parameters to obtain the highest signal-to-noise ratio.

In every camera system many noise sources exists, ${ }^{37}$ but for luminescence measurements we identify photon noise, dark noise, readout noise, and cooling fluctuations as the most dominant sources. In the following, we give a short review of the different noise sources. Note that the presented statistics of the noise sources are only valid if the analysis is carried out in signal electrons (and not in digital units).

\section{A. Signal electrons and noise}

The photon noise is an elementary characteristic of the discrete nature of the light and is described as a variation of the emitted photons in time. It is described with the Poisson statistics and the variance of $N_{\mathrm{ph}}$ photons is $N_{\mathrm{ph}}$. These photons are converted to signal electrons with the sensor's quantum efficiency $\eta(\lambda), N_{\mathrm{s}}=\eta N_{\mathrm{ph}}$. Hence, the statistical noise of the photons is directly assigned to the signal electrons. Thus, the variance $\sigma_{\mathrm{s}}^{2}$ of $N_{\mathrm{s}}$ signal electrons is

$$
\sigma_{\mathrm{s}}^{2}=N_{\mathrm{s}}=\Phi_{\mathrm{s}} t_{\mathrm{exp}},
$$

where we used the signal electron flux $\Phi_{\mathrm{s}}$, describing the generated signal electrons per time and per pixel, and the exposure time $t_{\exp }$ of the sensor.

\section{B. Dark electrons and noise}

Thermal stimulation within the sensor generates free electrons $N_{\text {drk }}$ which are indistinguishable from the signal electrons and thus wrongly counted as signal. Besides, the same Poisson statistics applies to those thermally generated electrons and thus their variance using the dark electron flux

$$
\begin{aligned}
& \Phi_{\mathrm{drk}} \text { is } \\
& \qquad \sigma_{\mathrm{drk}}^{2}=N_{\mathrm{drk}}=\Phi_{\mathrm{drk}} t_{\mathrm{exp}} .
\end{aligned}
$$

\section{Readout noise}

The readout noise $\sigma_{\text {ro }}$ is added to each pixel due to the readout electronics. The readout electronics handles the amplification of the signal electrons, the conversion into a voltage and its digitization to digital units.

\section{Cooling fluctuations}

Fluctuations of the sensor's cooling cause an additional noise source $\sigma_{\text {tmp }}$ because the amount of dark current mainly depends on the sensor's temperature. Temperature fluctuations vanish in the used Si cameras because of the very deep cooling and the large band gap. Instead, for the used InGaAs camera, $\sigma_{\mathrm{tmp}}$ is a quite substantial noise source. We estimate $\sigma_{\text {tmp }}$ by measuring the temperature variance and the relation of temperature and dark signal.

For a modeling of the signal-to-noise ratio the total noise of the image acquisition has to be determined from the presented parameters. Since the noise sources are not correlated, the variances simply add up to the total variance. For the total noise $\sigma_{\mathrm{df}}$ of the dark-frame subtracted image,

$$
\sigma_{\mathrm{df}}^{2}=\sigma_{\mathrm{s}}^{2}+2 \sigma_{\mathrm{drk}}^{2}+2\left(\sigma_{\mathrm{ro}}^{2}+\sigma_{\mathrm{tmp}}^{2}\right),
$$

the dark noise and the readout noise have to be counted twice as these noise sources are contained in the illuminated as well as in the dark image. The noise of the dark-frame subtracted image is thus bigger than the noise of the single image. However, the subtraction of the dark frame is substantially necessary for the quantitative analysis of luminescence images since it removes the dark current and remaining stray light from the signal.

Equation (7) holds for sensors without amplifying gain. For electron multiplying CCD sensors, another noise source comes into play which is called excess noise $F$ and is a result of fluctuations of the electron multiplier gain $g .{ }^{38}$ This excess noise effects also other detected electron charges except the readout noise. Therefore, for EMCCD sensors Eq. (7) becomes ${ }^{39}$

$$
\sigma_{\mathrm{df}}^{2}=g^{2} F^{2}\left(\sigma_{\mathrm{s}}^{2}+2 \sigma_{\mathrm{drk}}^{2}+2 \sigma_{\mathrm{tmp}}^{2}\right)+2 \sigma_{\mathrm{ro}}^{2} .
$$

The excess noise $F$ can be well estimated ${ }^{39}$ with $\sqrt{2}$ and the gain $g$ depends on the specific camera settings. In the following Eq. (8) will be used for the total noise; the nonamplifying case is obtained using $g=1$ and $F=1$.

The presented setup allows to average multiple images $M$ times. This results in a smaller standard deviation of the mean of

$$
u=\frac{\sigma_{\mathrm{df}}}{\sqrt{M}} .
$$




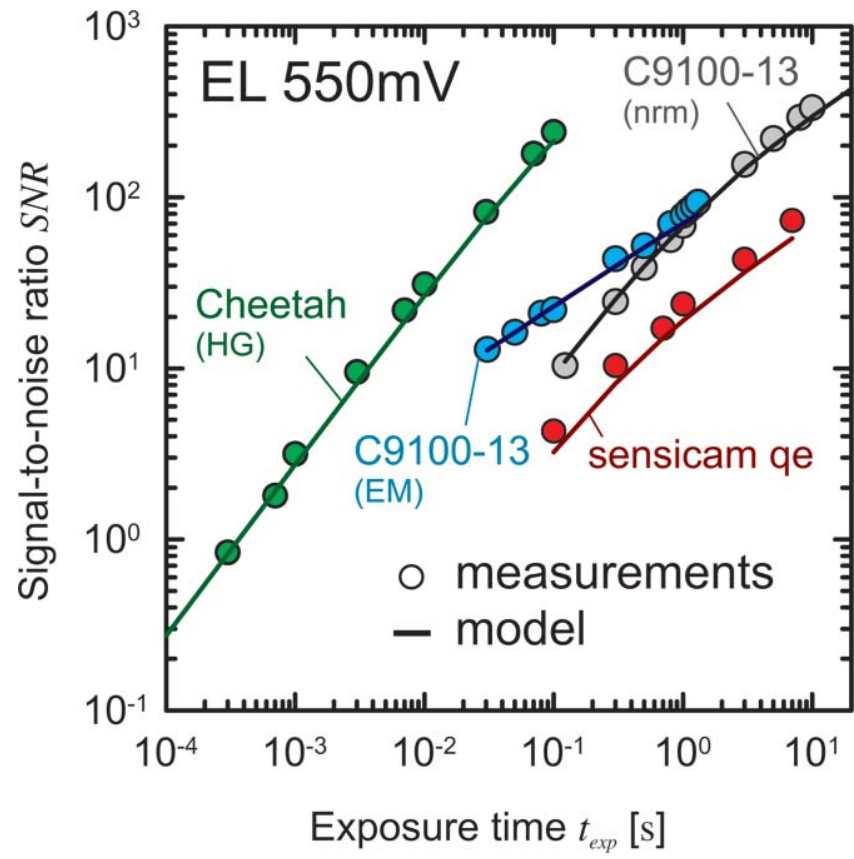

FIG. 6. (Color online) Calculated (lines) and measured (points) SNR curves for the Sensicam QE, the C9100-13 and the Cheetah camera for the same luminescence intensity of a solar cell hold at $550 \mathrm{mV}$. The resulting signals were averaged 20 times.

Putting Eq. (8) and Eq. (9) into Eq. (4) gives the signal-tonoise ratio

$$
\begin{aligned}
\mathrm{SNR}= & \sqrt{M} \Phi_{\mathrm{s}} g t_{\mathrm{exp}}\left(F^{2} g^{2}\left(\Phi_{\mathrm{s}}+2 \Phi_{\mathrm{drk}}\right) t_{\mathrm{exp}}\right. \\
& \left.+2 \sigma_{\text {ro }}^{2}+F^{2} g^{2} 2 \sigma_{\mathrm{tmp}}^{2}\right)^{-1 / 2} .
\end{aligned}
$$

For each camera type we experimentally determine the parameters $\sigma_{\mathrm{ro}}, \sigma_{\mathrm{tmp}}, \Phi_{\mathrm{drk}}$, and $\Phi_{\mathrm{s}}$ for the same source of luminescence. The results are listed in Table I. Note that the InGaAs-Camera (Cheetah) has a much higher dark electron flux than the Si-cameras. Using Eq. (10) we can thus calculate the expected SNR for each exposure time and luminescence intensity and compare the calculation with directly measured SNR values. Figure 6 shows the results for all three cameras. A good agreement over a wide range of exposure times demonstrates the validity of the presented model and of the determined parameters.

The question arises which camera settings (long exposure time or often averaging) to choose to obtain the best signal-tonoise ratio in a total measurement time $T=2 M t_{\text {exp }}$. Using Eq. (10) we deduce that due to the readout- and offset-noise a long exposure time always gives a higher SNR than averaging more often with a shorter exposure time. This is exemplary demonstrated in Fig. 7. Both images are taken for the same solar cell at the same operation conditions. The total measurement time $T=200 \mathrm{~s}$ was held fixed. The parameters exposure time and number of averages are set for Fig. 7(a) to $1 \mathrm{~s}$ and 100 times and for Fig. 7(b) to $100 \mathrm{~s}$ and 1 time. It can be clearly seen that the image quality of Fig. 7(b) is better which corresponds to a higher signal-to-noise ratio of 12.0 [compare to 2.9 for Fig. 7(a)].

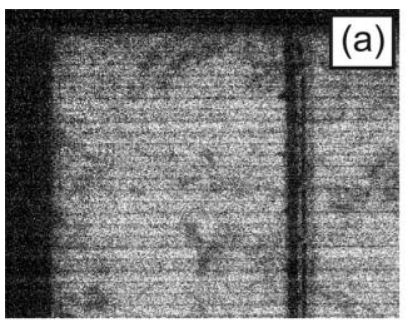

$t_{\text {exp }}=1 \mathrm{~s}, M=100$

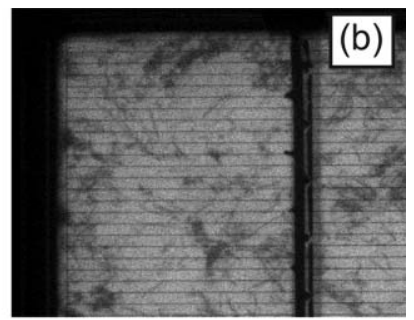

$t_{\exp }=100 \mathrm{~s}, M=1$

FIG. 7. Comparison of resulting images with a constant total measuring time. We obtain for image (a) a SNR of 2.9 and for images (b) a SNR of 12.0. The images are captured using the Sensicam QE silicon CCD camera.

More generally, since the exposure time is limited by the full well capacity $N_{\mathrm{FW}}$, we state that for a certain luminescence intensity and dark current the exposure time giving the highest SNR is

$$
t_{\mathrm{exp}}^{\max }=\frac{h \cdot N_{\mathrm{FW}}}{\Phi_{\mathrm{s}}+\Phi_{\mathrm{drk}}} .
$$

The factor $h$ accounts for the optimum range of each camera to maintain a good linear relationship of impinging photons and resulting signal. Since it is recommended to not fully saturate the pixel we usually use $h \approx 0.75$ for the cameras used in our setup. Using $t_{\exp }^{\max }$ and $M^{\max }=T / 2 / t_{\exp }^{\max }$ in Eq. (10) we obtain

$$
\begin{aligned}
& \mathrm{SNR}^{\max }=\sqrt{T} \Phi_{\mathrm{s}} g \\
& \times \sqrt{\frac{t_{\mathrm{exp}}^{\max }}{2 F^{2} g^{2}\left(\Phi_{\mathrm{s}}+2 \Phi_{\mathrm{drk}}\right) t_{\mathrm{exp}}^{\max }+4 \sigma_{\mathrm{ro}}^{2}+4 F^{2} g^{2} \sigma_{\mathrm{tmp}}^{2}}},
\end{aligned}
$$

which holds if $M^{\max }$ is a whole number and larger than or equal to 2 . The maximum possible SNR of the camera is thus proportional to the square-root of the total measurement time $T$ but depends on the particular camera settings and the luminescence intensity. Thus, different cameras can be better suited for low intensity than for high intensity and vice versa.

For the cameras investigated in this paper, Fig. 8 shows the dependence of the SNR of each camera to a certain luminescence intensity. The lines in this figure correspond to simulated values while the points are measured values. For a better understanding and comparison, the luminescence intensities are expressed additionally as a local voltage. Note that the correlation to the local voltage does only hold for the specific setup used.

In Fig. 8, a good agreement between the measured and simulated data is obtained. As expected, the SNR of the InGaAs camera is about one magnitude higher than the SNR of the Si cameras investigated in. But looking at camera costs per SNR achievable we can't identify a clear winner. It also becomes clear in Fig. 8, that the suitability of the different cameras and different camera modes to measure a high-quality image of the luminescence emission depends on the amount of captured luminescence. The EM mode of the Hamamatsu C9100-13 is better suited to very low signal measurements while the normal camera mode gives a better SNR for high 


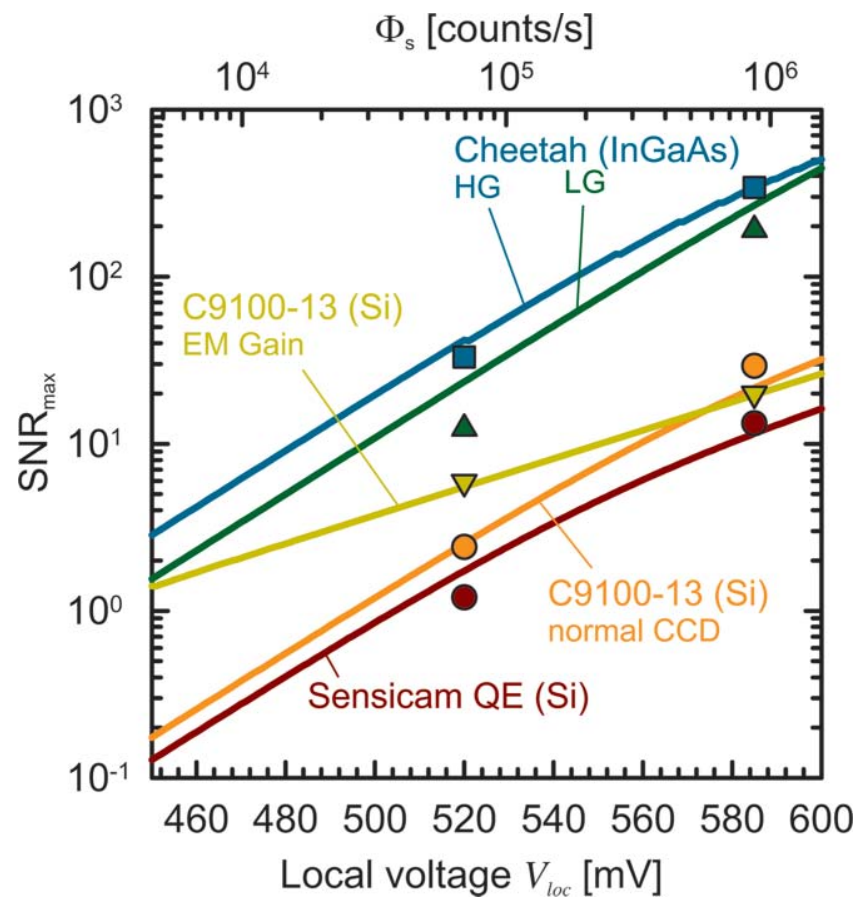

FIG. 8. (Color online) Comparison of the maximum signal-to-noise ratio for different cameras. The total measurement time is $\mathrm{T}=1 \mathrm{~s}$. The lines give the simulated signal-to-noise ratio and the points correspond to measured values.

signals. This behavior is observed in Fig. 8 in the simulated as well as in the measured data.

\section{CONCLUSION}

We introduced and characterized a luminescence imaging setup allowing a quantitative analysis of the luminescence emission of silicon solar cells and wafers. Details were given on the components used in this setup including different cameras, optical filters for electro- and photo-luminescence, a semiconductor laser, and a power supply. We compared the applicability for luminescence imaging of three different types of cameras. These cameras are a silicon CCD camera, a back-illuminated silicon CCD camera comprising an electron multiplier (EM) gain and a CMOS InGaAs camera. For all three cameras the dominant noise sources, the photon noise, the dark noise, the read-out noise, and the noise of the temperature stabilization were determined. Using these values we analyzed the signal-to-noise ratio of each camera at different operation conditions. We showed, that the resulting SNR only depends on the intensity of the emitted luminescence photons if the optimum exposure time is chosen. The SNR of the InGaAs camera is about one to two magnitudes higher than the SNR of the Si cameras. Regarding the two silicon CCD cameras under investigation we obtained that the EM gain is only an advantage for samples under low excitation conditions but suffers from a lower SNR under higher excitation conditions.

\section{ACKNOWLEDGMENTS}

This work was funded by the Federal Ministry for the Environment, Nature Conservation and Nuclear Safety under Contract No. 0327661.
${ }^{1}$ I. Uchida, Jpn. J. Appl. Phys. 2, 561 (1963).

${ }^{2} \mathrm{~K}$. Penner, Electroluminescence from silicon devices-a tool for device and material characterization 49, C4 (1988).

${ }^{3}$ G. Livescu, M. Angell, and J. Filipe, J. Electron. Mater. 19, 937 (1990).

${ }^{4}$ T. Fuyuki, H. Kondo, Y. Kaji, T. Yamazaki, Y. Takahashi, and Y. Uraoka, in Proceedings of the 31st Photovoltaic Specialists Conference, Lake Buena Vista, FL (IEEE, New York, 2005), pp. 1343-1345.

${ }^{5}$ T. Trupke, R. Bardos, M. Abbott, F. W. Chen, J. Cotter, and A. Lorenz, in Proceedings of the Fourth World Conference on Photovoltaic Energy Conversion, Waikoloa, Hawaii (IEEE, New York, 2006), pp. 928-931.

${ }^{6}$ M. Kasemann, W. Kwapil, M. Schubert, H. Habenicht, B. Walter, M. The, S. Kontermann, S. Rein, O. Breitenstein, J. Bauer, A. Lotnyk, B. Michl, H. Nagel, A. Schütt, J. Carstensen, H. Föll, T. Trupke, Y. Augarten, H. Kampwerth, R. Bardos, S. Pingel, J. Berghold, W. Warta, and S. Glunz, in Proceedings of the 33rd Photovoltaic Specialists Conference, San Diego, $C A$ (IEEE, New York, 2008).

${ }^{7}$ K. Bothe, P. Pohl, J. Schmidt, T. Weber, P. Altermatt, B. Fischer, and R. Brendel, in Proceedings of the 21st European Photovoltaic Solar Energy Conference, Dresden, Germany (WIP, Munich, 2006), pp. 597-600.

${ }^{8}$ M. Glatthaar, J. Haunschild, R. Zeidler, M. Demant, J. Greulich, B. Michl, W. Warta, S. Rein, and R. Preu, J. Appl. Phys. 108, 014501 (2010).

${ }^{9}$ T. Trupke, E. Pink, R. Bardos, and M. Abbott, Appl. Phys. Lett. 90, 093506 (2007).

${ }^{10}$ K. Ramspeck, K. Bothe, D. Hinken, B. Fischer, J. Schmidt, and R. Brendel, Appl. Phys. Lett. 90, 153502 (2007).

${ }^{11}$ D. Hinken, K. Ramspeck, K. Bothe, B. Fischer, and R. Brendel, Appl. Phys. Lett. 91, 182104 (2007).

${ }^{12}$ H. Kampwerth, T. Trupke, J. W. Weber, and Y. Augarten, Appl. Phys. Lett. 93, 02102 (2008).

${ }^{13}$ M. Glatthaar, J. Haunschild, M. Kasemann, J. Giesecke, W. Warta, and S. Rein, Phys. Status Solidi (RRL) 4, 13 (2010).

${ }^{14}$ P. Würfel, T. Trupke, T. Puzzer, E. Schaffer, W. Warta, and S. Glunz, J. Appl. Phys. 101, 123110 (2007).

${ }^{15}$ K. Bothe, D. Hinken, K. Ramspeck, B. Fischer, and R. Brendel, in Proceedings of the 22nd European Photovoltaic Solar Energy Conference, Milan, Italy (WIP, Munich, 2007), pp. 1673-1677.

${ }^{16}$ A. Helbig, T. Kirchartz, and U. Rau, in Proceedings of the 23rd European Photovoltaic Solar Energy Conference (WIP, Munich, 2008), pp. 426429.

${ }^{17}$ J. Giesecke, M. Kasemann, and W. Warta, J. Appl. Phys. 106, 014907 (2009).

${ }^{18}$ D. Hinken, K. Bothe, K. Ramspeck, S. Herlufsen, and R. Brendel, J. Appl. Phys. 105, 04516 (2009).

${ }^{19}$ D. Hinken, K. Bothe, K. Ramspeck, S. Herlufsen, and R. Brendel, in Proceedings of the 24th European Photovoltaic Solar Energy Conference, Hamburg, Germany (WIP, Munich, 2009), pp. 1082-1085.

${ }^{20} \mathrm{O}$. Breitenstein, J. Bauer, T. Trupke, and R. Bardos, Prog. Photovoltaics 16, 325 (2008).

${ }^{21}$ M. Kasemann, D. Grote, B. Walter, W. Kwapil, T. Trupke, Y. Augarten, R. Bardos, E. Pink, M. Abbott, and W. Warta, Prog. Photovoltics 16, 297 (2008).

${ }^{22}$ M. Bail, J. Kentsch, R. Brendel, and M. Schulz, in Proceedings of the 28th IEEE Photovoltaic Specialists Conference (IEEE, New York, 2000), pp. 99-103.

${ }^{23}$ M. The, M. Schubert, and W. Warta, in Proceedings of the 22nd European Photovoltaic Solar Energy Conference, Milan, Italy (WIP, Munich, 2007), pp. 354-359.

${ }^{24}$ T. Trupke, R. A. Bardos, M. C. Schubert, and W. Warta, Appl. Phys. Lett. 89, 44107 (2006).

${ }^{25}$ T. Trupke, R. A. Bardos, and M. D. Abbott, Appl. Phys. Lett. 87, 84102 (2005).

${ }^{26}$ R. Sinton and A. Cuevas, Appl. Phys. Lett. 69, 2510 (1996).

${ }^{27}$ D. Macdonald, J. Tan, and T. Trupke, J. Appl. Phys. 103, 073710 (2008).

${ }^{28} \mathrm{~T}$. Trupke and R. Bardos, in Proceedings of the 18th Workshop on Crystalline Silicon Solar Cells \& Modules, Vail, USA (NREL, Golden, 2008).

${ }^{29}$ S. Herlufsen, J. Schmidt, D. Hinken, K. Bothe, and R. Brendel, Phys. Status Solidi (RRL) 1-3, 245 (2008). 
${ }^{30}$ S. Herlufsen, K. Ramspeck, D. Hinken, A. Schmidt, J. Müller, K. Bothe, J. Schmidt, and R. Brendel, in Proceedings of the 25th European Photovoltaic Solar Energy Conference, Valencia, Spain (WIP, Munich, 2010).

${ }^{31}$ D. Kiliani, G. Micard, B. Raabe, and G. Hahn, in Proceedings of the 25th European Photovoltaic Solar Energy Conference, Valencia, Spain (WIP, Munich, 2010).

${ }^{32}$ K. Bothe, K. Ramspeck, D. Hinken, C. Schinke, J. Schmidt, S. Herlufsen, R. Brendel, J. Bauer, J.-M. Wagner, N. Zakharov, and O. Breitenstein, J. Appl. Phys. 106, 104510 (2009).

${ }^{33}$ J. Giesecke, M. Kasemann, M. Schubert, B. Michl, M. The, W. Warta, and P. Würfel, in Proceedings of the 23th European Photovoltaic Solar Energy Conference, Valencia, Spain (WIP, Munich, 2008).
${ }^{34}$ K. Bindra, R. Chari, V. Shukla, A. Singh, S. Ida, and S. Oak, J. Opt. A, Pure Appl. Opt. 1, 73 (1999).

${ }^{35}$ M. Abbott, R. Bardos, T. Trupke, K. Fisher, and E. Pink, J. Appl. Phys. 102, 044502 (2007)

${ }^{36}$ M. Seibt, R. Khalil, V. Kveder, and W. Schröter, Appl. Phys. A 96, 235 (2009).

${ }^{37}$ J. Janesick, Scientific Charge Coupled Devices, SPIE Press Monograph Vol. PM83 (2001).

${ }^{38}$ J. Hynecek and T. Nishiwaki, IEEE Trans. Electron Devices 50, 239 (2003).

${ }^{39}$ M. Robbins and B. Hadwen, IEEE Trans. Electron Devices 50, 1227 (2003). 